\title{
High levels of reactive oxygen species in pancreatic necrotic fluid of patients with walled-off pancreatic necrosis
}

\author{
Lukasz Rojek ${ }^{1}$, Areta Hebanowska², Magdalena Stojek ${ }^{1}$, Mateusz Jagielski ${ }^{1}$, Elzbieta Goyke ${ }^{2}$, \\ Sylwia Szrok-Jurga ${ }^{2}$, Marian Smoczynski ${ }^{1}$, Julian Swierczynski ${ }^{3}$, Tomasz Sledzinski ${ }^{4}$, Krystian Adrych $^{1}$ \\ ${ }^{1}$ Department of Gastroenterology and Hepatology, Faculty of Medicine, Medical University of Gdansk, Gdansk, Poland \\ 2Department of Biochemistry, Faculty of Medicine, Medical University of Gdansk, Gdansk, Poland \\ ${ }^{3}$ State School of Higher Vocational Education, Koszalin, Poland \\ ${ }^{4}$ Department of Pharmaceutical Biochemistry, Faculty of Pharmacy, Medical University of Gdansk, Gdansk, Poland
}

Gastroenterology Rev 2021; 16 (1): 56-61 DOI: https://doi.org/10.5114/pg.2020.95948

Key words: reactive oxygen species, walled-off pancreatic necrosis.

Address for correspondence: Prof. Krystian Adrych, Department of Gastroenterology and Hepatology, Faculty of Medicine, Medical University of Gdansk, Poland, e-mail: krystian.adrych@gumed.edu.pl

\begin{abstract}
Introduction: Walled-off pancreatic necrosis (WOPN) is a life-threatening, late complication of acute pancreatitis, in which a fluid collection containing necrotic material is formed. Infection of the fluid collection significantly increases the mortality of patients with WOPN.

Aim: To examine the levels of oxidative stress markers in the pancreatic necrotic fluid (PNF) and serum of patients with sterile and infected WOPN.

Material and methods: Thirty-three adult patients with sterile WOPN and 14 with infected WOPN, as well as 31 patients with mild AP, were included in this study. Concentrations of oxidative stress markers (8-isoprostane, protein carbonyl groups, and 8-hydroxyguanine) were measured in the PNF and serum of patients with sterile and infected WOPN.

Results: High concentrations of all measured oxidative stress markers in PNF, but not in serum, were detected in patients with WOPN. Additionally, oxidative stress markers in PNF were significantly increased in patients with infected as compared to sterile WOPN. The serum high sensitive C-reactive protein (hSCRP) concentrations showed the highest correlation with PNF oxidative stress marker levels. Receiver operating characteristics (ROC) curve analysis confirmed that serum hsCRP could be a good predictor of WOPN infection.

Conclusions: Oxidative stress is associated with WOPN development; infection of PNF worsens the course of WOPN, possibly via increased production of reactive oxygen species; and serum hsCRP concentrations seem to be a good, noninvasive indicator of PNF infection.
\end{abstract}

\section{Introduction}

Necrotising acute pancreatitis accounts for $10-15 \%$ of cases of acute pancreatitis (AP) and is a potentially life-threatening condition due to its late complications such as post-inflammatory pancreatic fluid collections. A collection of necrotic material arising from peripancreatic or pancreatic tissue that has matured, typically within 4 weeks, and developed a well-defined fibrous wall is termed walled-off pancreatic necrosis (WOPN) $[1,2]$. WOPN may be sterile or infected by bacteria and/ or fungi $[3,4]$. Infection significantly increases mortali- ty and morbidity [5]. Diagnosis and confirmation of infected WOPN requires puncture of the fluid collection - an invasive procedure carrying a risk of introducing iatrogenic infection into a sterile WOPN [6]. Therefore, it would be useful to find reliable serum parameter(s) indicating an infection of WOPN without the need to perform an invasive procedure.

Oxidative stress plays an important role in the pathogenesis and progression of AP [7, 8]. Chronic stress induces ischaemia of the pancreatic tissue followed by reperfusion, accompanied by hypoxia and reoxygenation, which eventually lead to formation of 
reactive oxygen species (ROS). It may also attract leukocytes, which release additional ROS in a respiratory burst. Increased immature granulocyte count at the beginning of acute pancreatitis was observed in patients with severe, but not mild, course of the disease [9]. All those events are part of the very early stages of this process [10]. No studies have to date investigated oxidative stress markers in patients with WOPN. Serum protein carbonyl groups, isoprostanes and 8-hydroxyguanine, products of oxidation of proteins, polyunsaturated fatty acids, and nucleic acids, respectively, are often examined as oxidative stress markers [11].

\section{Aim}

The aim of this study was to determine the serum concentrations of 8-isoprostane, 8-hydroxyguanine, and protein carbonyl groups in patients with AP and sterile and infected WOPN as well as the pancreatic necrotic fluid (PNF) concentrations of these markers in patients with sterile and infected WOPN. Moreover, we tried to find serum parameters that would correlate with PNF concentrations of oxidative stress markers and serve as a potential noninvasive predictor of WOPN infection.

\section{Material and methods}

The study included 47 adult patients with WOPN admitted to the Department of Gastroenterology and Hepatology, Medical University of Gdansk. Thirty-three of them had sterile WOPN, and 14 had infected WOPN. The mean age of patients in each group was $48 \pm 12$ and $53 \pm 17$ years, respectively. The WOPN was identified according to the definitions set forth in the Revision of the Atlanta classification [6]. The presence of infection was presumed based on clinical signs (such as deterioration of general condition or fever) or the presence of extraluminal gas in the pancreatic or peripancreatic tissue on contrast-enhanced computed tomography. PNF samples were obtained during the first step of endoscopic treatment of symptomatic WOPN as described previously [12-14]. Infection of WOPN was confirmed by culture of PNF obtained from image-guided fine-needle aspiration in 14 patients. Pathogens detected in the infected WOPN fluid included the following: Escherichia coli, Streptococcus, Peptostreptococcus, Prevotella, Enterococcus, Enterobacter, Klebsiella, Pseudomonas, Staphylococcus, Morganella, Proteus, Acinetobacter, Bacteroides, and Candida. In 9 patients, WOPN was infected with multiple pathogens. Blood and PNF samples were obtained during the first endoscopic procedure performed as part of WOPN endotherapy.

The study also included 31 adult patients with AP admitted to the Department of Gastroenterology and Hepatology, Medical University of Gdansk. All of them had a mild form of the disease. The diagnosis of acute pancreatitis and the severity of the disease were established according to the Atlanta classification 2012 [6]. The mean age of this group of patients was $53 \pm 16$ years. Blood samples in this group were obtained between the first and third day of hospitalisation.

Thirty-five healthy male volunteers (aged $46 \pm 11$ years) from the same demographic group as the patients with AP and WOPN, who received annual health examinations and reported only occasional alcohol consumption, formed the control group.

The study was performed in accordance with the Declaration of Helsinki of the World Medical Association and was approved by the Medical University of Gdansk Ethics Committee. All patients signed an informed consent form for this investigation.

Blood samples were obtained from all subjects by venipuncture and centrifuged within 30-45 min at $3000 \times \mathrm{g}$ for $20 \mathrm{~min}$. Serum samples were stored at $-80^{\circ} \mathrm{C}$ until analysis. In patients with WOPN, PNF was obtained during the drainage procedure by needle aspiration, several samples collected into $2 \mathrm{ml}$ Eppendorf tubes and then immediately frozen in liquid nitrogen. The remaining fluid was transported immediately to the Central Medical Laboratory of the Medical University of Gdansk and to the Department of Biochemistry of the Medical University of Gdansk for further analysis. Biochemical analysis was performed on supernatant obtained after centrifugation of PNF (20000 $\times \mathrm{g}$ for $20 \mathrm{~min}$ ). Standard laboratory parameters in blood were measured at the Central Medical Laboratory of the Medical University of Gdansk.

Serum protein carbonyl groups were assayed using a modified method by Levine [15].

Serum and PNF 8-hydroxyguanine and 8-isoprostane concentrations were measured by commercial immunoassay (Cayman Chemical) according to the manufacturer's instructions.

Serum and PNF free fatty acid (FFA) concentration was measured by the enzymatic, colorimetric method according to the protocol supplied by Wako Chemicals $\mathrm{GmbH}$ (Germany).

\section{Statistical analysis}

Descriptive statistics were used to summarise baseline demographic data. All data were expressed as mean $\pm \mathrm{SEM}$. For normally distributed data the statistical significance of differences between means of the various biochemical parameters was estimated by using parametric (Student's $t$ ) test for comparison between two groups or one-way ANOVA followed by post-hoc for comparison of more than two groups. The data without normal distribution were analysed by non- 
parametric (U Mann-Whitney, Wilcoxon) tests or ANOVA on ranks, respectively. The diagnostic ability of selected parameters to predict WOPN infection was performed based on receiver operating characteristic (ROC) curve analysis. A $p$-value of $<0.05$ was considered statistically significant for all analyses. Statistical analysis was performed using SigmaPlot software.

\section{Results}

Standard laboratory abnormalities found in patients with AP, sterile and infected WOPN are presented in Table I.

There was a striking increase in oxidative stress markers in pancreatic necrotic fluid (PNF), with 8-isoprostane and carbonyl groups significantly elevated compared to their serum concentrations (Table II). The concentrations of 8-isoprostane, carbonyl groups, and 8-hydroxyguanine were also approximately 3-4-fold higher in PNF of infected compared to sterile WOPN (Table II). However, serum concentrations of carbonyl groups, 8-isoprostane, and 8-hydroxyguanine were not elevated in patients with AP nor in those with WOPN, with serum 8-hydroxyguanine concentrations even slightly decreased in AP and infected WOPN (Table II).

Other remarkable findings included significantly increased FFA in the pancreatic necrotic fluid compared to serum concentrations, with no statistically significant differences between sterile and infected WOPN (Table II).
Patients with AP and WOPN, especially infected WOPN, had increased serum high-sensitive C-reactive protein (hsCRP) concentrations. White blood cells (WBC) were significantly increased only in patients with AP and infected WOPN (Table II). Among the parameters examined, serum hsCRP concentrations had the highest correlation with PNF carbonyl groups, 8-hydroxyguanine and 8-isoprostane (Table III). Also, the ROC curve analysis showed that serum hsCRP could be a good predictor for WOPN infection (area under curve (AUC) = 0.86; $p<0.01$; 95\% confidence interval (Cl): 0.760-0.967, Figure $1 \mathrm{~A}$ ). Moreover, a positive correlation was also found between serum 8-isoprostane and PNF carbonyl groups as well as between white blood cell count and PNF carbonyl groups (Table III). Among these variables only WBC was a significant predictor for WOPN infection, but much weaker than hsCRP (AUC $=0.61 ; p=0.03$; 95\% Cl: 0.423-0.797, Figure $1 \mathrm{~B}$ ).

\section{Discussion}

Reactive oxygen species play an important role in the pathogenesis of inflammation and tissue injury. In the setting of oxidative stress, when ROS production and levels exceed those of antioxidants, tissue damage occurs [16]. The major finding of this study is the presence of severely increased levels of oxidative stress products in PNF of patients with WOPN. It is generally believed that oxidative stress typically develops in ear-

Table I. Selected serum or blood biochemical parameters in respective subgroups. Data presented as mean \pm SEM

\begin{tabular}{|c|c|c|c|c|}
\hline Parameter & Control & Acute pancreatitis & Sterile WOPN & Infected WOPN \\
\hline Amylase [U/I] & $50 \pm 6.8$ & $1205 \pm 622^{*}$ & $127 \pm 34^{*}$ & $117 \pm 26$ \\
\hline Lipase [U/I] & $24.6 \pm 3.4$ & $603 \pm 114^{*}$ & $103 \pm 33^{*}$ & $95.0 \pm 28^{*}$ \\
\hline$\gamma$-glutamyl transpeptidase [U/I] & $34 \pm 3$ & $355 \pm 89^{*}$ & $133 \pm 49^{*}$ & $254 \pm 78^{\star \#}$ \\
\hline Alkaline phosphatase [U/I] & $67 \pm 4.8$ & $158 \pm 23^{\star}$ & $139 \pm 35^{*}$ & $180 \pm 38^{*}$ \\
\hline Alanine transaminase [U/I] & $21 \pm 1.6$ & $115 \pm 26^{\star}$ & $28.7 \pm 7.5$ & $24.2 \pm 8.2$ \\
\hline Aspartate transaminase [U/I] & $24 \pm 1.34$ & $93.2 \pm 22^{*}$ & $27.6 \pm 6.8$ & $33.8 \pm 14^{*}$ \\
\hline Glucose $[\mathrm{mg} / \mathrm{dl}]$ & $91.8 \pm 2.0$ & $121 \pm 11^{\star}$ & $112 \pm 7.0^{\star}$ & $121 \pm 14^{\star}$ \\
\hline Triacylglycerol [mg/dl] & $87 \pm 5.4$ & $276 \pm 82^{\star}$ & $121 \pm 8.7^{\star}$ & $193 \pm 33^{\star \#}$ \\
\hline Total cholesterol [mg/dl] & $198 \pm 5.2$ & $216 \pm 22$ & $179 \pm 10^{\star}$ & $141 \pm 10^{\star \#}$ \\
\hline Total protein [g/l] & $76.5 \pm 0.52$ & $61.4 \pm 1.2^{*}$ & $69.3 \pm 1.6^{*}$ & $63.5 \pm 1.9^{* \#}$ \\
\hline Albumin $[g / l]$ & $44.7 \pm 0.74$ & $29.6 \pm 1.3^{*}$ & $34.0 \pm 1.6^{\star}$ & $22.5 \pm 1.1^{\text {*\# }}$ \\
\hline Haemoglobin [g/l] & $15.5 \pm 0.54$ & $13.7 \pm 0.22^{*}$ & $13.1 \pm 0.34^{*}$ & $10.3 \pm 0.48^{\star \#}$ \\
\hline Bilirubin [mg/dl] & $0.82 \pm 0.06$ & $3.0 \pm 0.46^{*}$ & $1.55 \pm 0.08^{*}$ & $0.65 \pm 0.09^{\star \#}$ \\
\hline Creatinine [mg/dl] & $0.80 \pm 0.02$ & $0.99 \pm 0.04^{*}$ & $0.78 \pm 0.03$ & $0.69 \pm 0.05$ \\
\hline
\end{tabular}

${ }^{*} P<0.05$ compared to controls; $P<0.05$ compared to sterile WOPN; WOPN-walled-off pancreatic necrosis. Haemoglobin was assayed in blood and all other parameters were assayed in serum of study subjects. 
Table II. Selected oxidative stress and some other biochemical parameters in PNF and serum or blood in respective subgroups. Data presented as mean \pm SEM

\begin{tabular}{|c|c|c|c|c|c|c|}
\hline \multirow[t]{2}{*}{ Parameter } & \multicolumn{4}{|c|}{ Serum or blood } & \multicolumn{2}{|c|}{ PNF } \\
\hline & Control & $\begin{array}{c}\text { Acute } \\
\text { pancreatitis }\end{array}$ & Sterile WOPN & Infected WOPN & Sterile WOPN & Infected WOPN \\
\hline 8-isoprostane [pg/ml] & $23.8 \pm 0.52$ & $21.3 \pm 3.3$ & $20.9 \pm 3.0$ & $25.2 \pm 10.4$ & $635 \pm 309^{*}$ & $2894 \pm 1152^{* \#}$ \\
\hline 8-hydroxyguanine $[\mathrm{pg} / \mathrm{ml}]$ & $6729 \pm 291$ & $5641 \pm 250^{*}$ & $6144 \pm 489$ & $4686 \pm 489^{\star}$ & $8298 \pm 865$ & $31688 \pm 8992^{\star \#}$ \\
\hline $\begin{array}{l}\text { Carbonyl groups } \\
\text { [nmol/mg protein] }\end{array}$ & $0.74 \pm 0.03$ & $0.97 \pm 0,03$ & $1.08 \pm 0.15$ & $0.79 \pm 0.04$ & $2.73 \pm 0.62^{*}$ & $7.50 \pm 1.3^{\star \#}$ \\
\hline $\mathrm{hsCRP}[\mathrm{mg} / \mathrm{l}]$ & $1.82 \pm 0.39$ & $155 \pm 17^{\star}$ & $34.2 \pm 11^{*}$ & $138 \pm 29^{*}$ & ND & ND \\
\hline WBC $\left[10^{9} / 1\right]$ & $6.57 \pm 0.39$ & $11.3 \pm 0.71^{\star}$ & $8.4 \pm 0.86$ & $9.7 \pm 1.0^{\star}$ & ND & ND \\
\hline $\mathrm{FFA}[\mathrm{mmol} / \mathrm{l}]$ & $0.17 \pm 0.02$ & $0.51 \pm 0.09^{\star}$ & $0.5 \pm 0.09^{*}$ & $0.5 \pm 0.06^{*}$ & $1.9 \pm 0.41^{*}$ & $2.9 \pm 0.91^{\star}$ \\
\hline
\end{tabular}

${ }^{*} P<0.05$ compared to controls; ${ }^{*} p<0.05$ compared to sterile PNF; ND - not determined, WOPN - walled-off pancreatic necrosis, PNF-pancreatic necrotic fluid, hSCRP - high sensitive C-reactive protein, FFA - free fatty acid, WBC - white blood cells. WBC was assayed in blood and all other parameters were assayed in serum of study subjects.

Table III. The correlation coefficients between selected serum or blood parameters and oxidative stress markers in pancreatic necrotic fluid

\begin{tabular}{lcccc} 
Variable & $\begin{array}{c}\text { Serum carbonyl } \\
\text { groups }\end{array}$ & $\begin{array}{c}\text { Serum } \\
\text { 8-hydroxyguanine }\end{array}$ & $\begin{array}{c}\text { Serum } \\
\text { 8-isoprostane }\end{array}$ & Serum hsCRP \\
\hline PNF carbonyl groups & -0.15 & -0.09 & $0.35^{*}$ & $0.49^{*}$ \\
\hline PNF 8-hydroxyguanine & -0.08 & 0.05 & 0.10 & $0.44^{*}$ \\
\hline PNF 8-isoprostane & -0.18 & 0.25 & -0.04 & 0.17 \\
\hline
\end{tabular}

*Statistically significant at $p<0.05$; PNF-pancreatic necrotic fluid, hSCRP-high-sensitivity C-reactive protein, WBC-white blood cells.

A

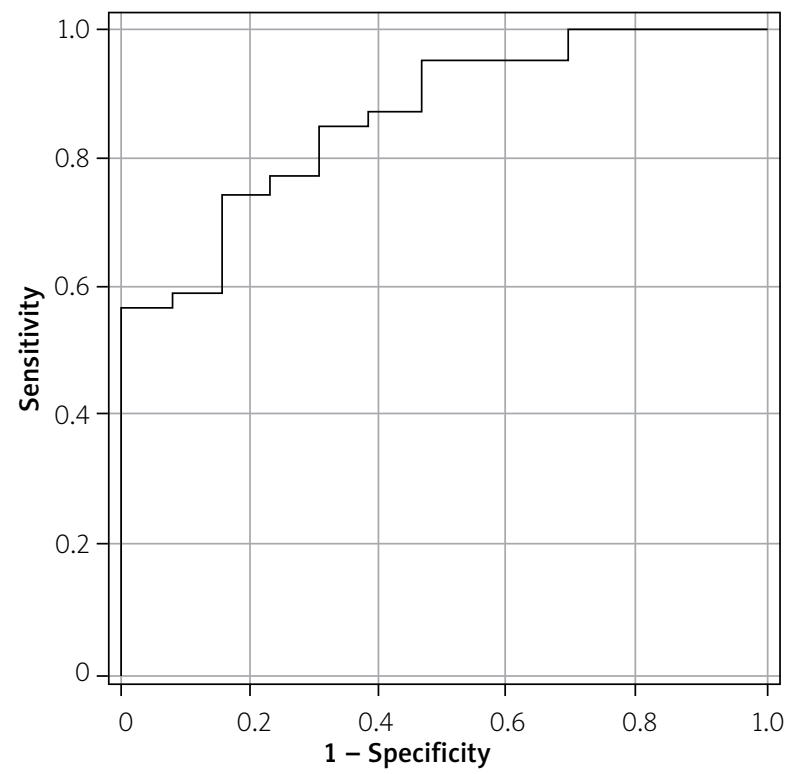

B

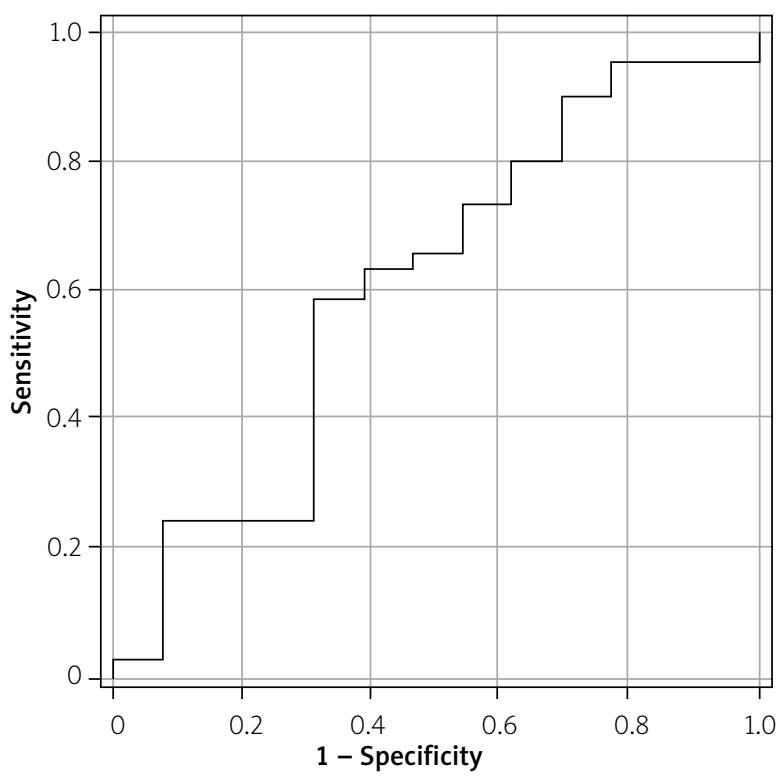

Figure 1. Receiver operating characteristic (ROC) curve analysis of serum high-sensitivity C-reactive protein (hsCRP) (A) and blood white blood cells (WBC) (B) as predictors for walled-off pancreatic necrosis (WOPN) infection. $\mathbf{A}$ - area under curve $(\mathrm{AUC})=0.86 ; p<0.01 ; 95 \%$ confidence interval $(\mathrm{Cl})$ : 0.760-0.967. B - AUC $=0.61 ; p=0.03 ; 95 \% \mathrm{Cl}: 0.423-0.797$ 
ly stages of AP [17]. The present study demonstrated increased levels of oxidative stress products, including 8-isoprostane, 8-hydroxyguanine, and carbonyl groups, in the PNF of patients with WOPN, a late complication of AP. This suggests that oxidative stress plays an important role not only in the pathogenesis of AP but also in the development of WOPN. Therefore, it is tempting to speculate that reducing the ROS production by antioxidant therapy may be clinically valuable for the supportive treatment of WOPN.

Of note, PNF concentrations of oxidative stress products were several times greater in patients with infected than sterile WOPN (Table II). This confirms the link between ROS production and microbial infection and implies that persistent infection increases ROS production by host cells. Interestingly, despite significantly higher levels of oxidative stress markers in the PNF of infected as compared with sterile WOPN, they were not reflected by their serum levels (Table II). One possible explanation could be limited permeability (or even impermeability) of the fibrous walls of the collection. In the early stages of AP oxidative stress is an intra-acinar as well as systemic process [17, 18]. By contrast, oxidative stress in PNF of patients with WOPN seems to be predominantly a local process, limited to the pancreas, surrounding tissues, and the contents of PNF collection. The damage of cells leads to the release of several enzymes and catabolites into PNF. In fact, our previous study showed that PNF obtained from patients with WOPN has an elevated activity of lactate dehydrogenase, malate dehydrogenase, and quantity of amino acids, which suggests ongoing necrosis [12]. The high concentrations of FFA in PNF of WOPN patients (Table II) reported in our present study could also be the result of cellular damage.

A new and important observation presented here is that oxidative stress is affected by infection, as demonstrated by the significant increase in the PNF levels of oxidative stress products in infected vs. sterile WOPN (Table II). Infection of WOPN may attract leukocytes and further increased local ROS production $[19,20]$. Increased local ROS production by polymorphonuclear neutrophils leads to further tissue injury.

Our study found no elevated serum markers of oxidative stress in patients with AP, unlike several other papers [8, 21-23]. The difference might be that our AP patients had mild disease and their serum samples were collected between the first and third day of hospitalisation, which may have affected the serum levels of oxidative stress markers.

The biochemical characteristics of WOPN have not yet been sufficiently studied, and increased knowledge in that regard could hopefully improve our understanding of its pathogenesis and treatment. It is especially import- ant to identify useful biomarkers of WOPN infection. At present, the microbiological diagnosis of PNF infection requires complicated endoscopic or percutaneous invasive procedures. These procedures carry the risk of introducing iatrogenic infection of a sterile WOPN. Therefore, they are only performed in cases of a high probability of WOPN infection and when endoscopic treatment for WOPN is indicated. Thus, there is a need to find reliable serum parameter(s) predicting WOPN infection without the need for invasive procedures. We found that an approximately four-fold increase in PNF oxidative stress markers is associated with a four-fold increase in serum hsCRP concentrations in patients with infected as compared with sterile WOPN (Table II). Moreover, serum hsCRP concentration correlates well with PNF markers of oxidative stress (Table III). Thus, one can assume that increased serum hsCRP concentrations could be a non-invasive marker of PNF infection in WOPN patients.

Our study has some limitations. First of all, it would be worthwhile to assess the biochemical characteristics of WOPN in a larger group of patients, especially with infected WOPN. However, this is not an easy task because drainage procedures (either via interventional radiology, endoscopy, or surgery) are rarely required. As already mentioned above, only approximately 5-15\% of patients with acute pancreatitis develop necrosis of the pancreatic parenchyma, the peripancreatic tissue, or both, and about $10-50 \%$ of those patients develop a superimposed bacterial infection $[6,24]$.

\section{Conclusions}

Our study revealed that WOPN is associated with highly elevated oxidative stress markers inside PNF collections. PNF concentrations of oxidative stress products were several times greater in patients with infected compared to sterile WOPN, possibly contributing to the serious course of this AP complication. Serum hSCRP concentration seems to be a good predictor of WOPN infection.

\section{Acknowledgments}

The study was supported by Medical University of Gdansk grants: ST-43, ST-41, ST-40, and Polpharma Starogard Gdanski, Poland.

\section{Conflict of interest}

The authors declare no conflict of interest.

\section{References}

1. Boumitri C, Brown E, Kahaleh M Necrotizing pancreatitis: current management and therapies. Clin Endosc 2017; 50: 357-65. 
2. Garber A, Frakes C, Arora Z, et al. Mechanisms and management of acute pancreatitis. Gastroenterol Res Pr 2018; 2018: 6218798.

3. Schmidt PN, Roug S, Hansen EF, et al. Spectrum of microorganisms in infected walled-off pancreatic necrosis - impact on organ failure and mortality. Pancreatology 2014; 14: 444-9.

4. Werge $M$, Roug S, Novovic $S$, et al. Fungal infections in patients with walled-off pancreatic necrosis. Pancreas 2016; 45: 1447-51.

5. Werge M, Novovic S, Schmidt PN, et al. Infection increases mortality in necrotizing pancreatitis: a systematic review and meta-analysis. Pancreatology 2016; 16: 698-707.

6. Banks PA, Bollen TL, Dervenis C, et al. Classification of acute pancreatitis - 2012: revision of the Atlanta classification and definitions by international consensus. Gut 2013; 62: 102-11.

7. $\mathrm{Yu} \mathrm{JH}, \mathrm{Kim} \mathrm{H}$. Oxidative stress and inflammatory signaling in cerulein pancreatitis. World J Gastroenterol 2014; 20: 17324-9.

8. Park BK, Chung JB, Lee JH, et al. Role of oxygen free radicals in patients with acute pancreatitis. World J Gastroenterol 2003; 9: 2266-9.

9. Lipiński M, Rydzewska G. Immature granulocytes predict severe acute pancreatitis independently of systemic inflammatory response syndrome. Gastroenterology Rev 2017; 12: 140-4.

10. Binker MG, Cosen-Binker LI. Acute pancreatitis: the stress factor. World J Gastroenterol 2014; 20: 5801-7.

11. Mayne ST. Antioxidant nutrients and chronic disease: use of biomarkers of exposure and oxidative stress status in epidemiologic research. J Nutr 2003; 133 Suppl: 933S-40S.

12. Rojek L, Smoczynski M, Stojek M, et al. Increased protein degradation as well as lactate and malate dehydrogenase activity in sterile and infected walled-off pancreatic necrosis. Pol Arch Med Wewn 2016; 126: 102-5.

13. Jagielski M, Smoczyński M, Adrych K. The role of transpapillary drainage in management of patients with pancreatic fluid collections and pancreatic duct disruption as a consequences of severe acute pancreatitis. Pancreatology 2017; 17: 30-1.

14. Jagielski M, Smoczyński M, Adrych K. The role of endoscopic treatment of pancreatic duct disruption in patients with walled-off pancreatic necrosis. Surg Endosc 2018; 32: 4939-52.

15. Levine RL, Garland D, Oliver CN, et al. Determination of carbonyl content in oxidatively modified proteins. Methods Enzymol 1990; 186: 464-78.

16. Mittal M, Siddiqui MR, Tran K, et al. Reactive oxygen species in inflammation and tissue injury. Antioxid Redox Signal 2014; 20: 1126-67.

17. Thareja S, Bhardwaj P, Sateesh J, et al. Variations in the levels of oxidative stress and antioxidants during early acute pancreatitis. Trop Gastroenterol 2010; 30: 26-31.

18. Seo JY, Kim H, Seo JT, et al. Oxidative stress induced cytokine production in isolated rat pancreatic acinar cells: effects of small-molecule antioxidants. Pharmacology 2002; 64: 63-70.

19. Bulkley GB. The role of oxygen free radicals in human disease processes. Surgery 1983; 94: 407-11.

20. Sanfey H, Bulkley GB, Cameron JL. The role of oxygen-derived free radicals in the pathogenesis of acute pancreatitis. Ann Surg 1984; 200: 405-13.
21. Rau B, Poch B, Gansauge F, et al. Pathophysiologic role of oxygen free radicals in acute pancreatitis: initiating event or mediator of tissue damage? Ann Surg 2000; 231: 352-60.

22. Winterbourn CC, Bonham MJD, Buss H, et al. Elevated protein carbonyls as plasma markers of oxidative stress in acute pancreatitis. Pancreatology 2003; 3: 375-82.

23. Hernandez V, Miranda M, Pascual I, et al. Malondialdehyde in early phase of acute pancreatitis. Rev Esp Enferm Dig 2011; 103: 563-9.

24. Portelli M, Jones CD. Severe acute pancreatitis: pathogenesis, diagnosis and surgical management. Hepatobiliary Pancreat Dis Int 2017; 16: 155-9.

Received: 23.10.2019

Accepted: 4.05.2020 